\title{
Baryonic Bd decays at Belle
}

\section{Min-Zu Wang ${ }^{* i}$}

National Taiwan University, Taiwan, R.O.C.

E-mail: mwangephys.ntu.edu.tw

Recent results of charmless baryonic $B$ decays from Belle are reported. These include the threebody decays of $B \rightarrow \Lambda \bar{\Lambda} h$ and $B^{+} \rightarrow p \bar{\Lambda} \rho^{0}$, and $B^{+} \rightarrow p \bar{\Lambda} \pi^{+} \pi^{-}$four-body decay, where $h$ stands for a light meson. This study is based on a $605 \mathrm{fb}^{-1}$ data sample, consisting of 657 million $B \bar{B}$ pairs, collected by the Belle detector at the KEKB asymmetric energy $e^{+} e^{-}(3.5$ on $8 \mathrm{GeV})$ collider.

European Physical Society Europhysics Conference on High Energy Physics, EPS-HEP 2009, July 16 - 222009

Krakow, Poland

\footnotetext{
* Speaker.

$\dagger$ on behalf of the Belle Collaboration
} 


\section{Introduction}

After the first observation of the charmless baryonic $B$ decays, namely, $B^{+} \rightarrow p \bar{p} K^{+}[1,2]$, many other three-body decay modes have been found but only very stringent upper limits being set for two-body decays [3]. These observed decays presumably proceed via the $b \rightarrow u(c)$ tree or the $b \rightarrow s(d)$ penguin diagrams. They may be used to search for direct $C P$ violation and test our theoretical understanding of rare decay processes involving baryons. One important experimental finding of these three-body decays is that the baryon-antibaryon mass distributions all peak near threshold. This feature attracts many theoretical investigations.

In this presentation, we will show a recent work [4] on the study of charmless three-body decays $B \rightarrow \Lambda \bar{\Lambda} h$, where $h$ stands for $\pi^{+}, K^{+}, K^{0}, K^{*+}$, or $K^{* 0}$. We will also report the search result for four-body charmless baryonic decay $B^{+} \rightarrow p \bar{\Lambda} \pi^{+} \pi^{-}$. In order to investigate the threshold enhancement effect, we study partial branching fractions as a function of the baryon-antibaryon mass. The data sample used in this study is about $605 \mathrm{fb}^{-1}$ which contains $657 \times 10^{6} B \bar{B}$ pairs collected with the Belle detector [5] on the $\Upsilon(4 \mathrm{~S})$ resonance at the KEKB asymmetric energy $e^{+} e^{-}$ (3.5 and $8 \mathrm{GeV}$ ) collider [6].

\section{Results of $B \rightarrow \Lambda \bar{\Lambda} h$}

We use two kinematic variables in the center of mass $(\mathrm{CM})$ frame to identify the reconstructed $B$ meson candidates: the beam energy constrained mass $M_{\mathrm{bc}}=\sqrt{E_{\mathrm{beam}}^{2}-p_{B}^{2}}$, and the energy difference $\Delta E=E_{B}-E_{\text {beam }}$, where $E_{\text {beam }}$ is the beam energy, and $p_{B}$ and $E_{B}$ are the momentum and energy, respectively, of the reconstructed $B$ meson. We perform an unbinned extended likelihood fit using the information from $M_{\mathrm{bc}}$ and $\Delta E$ (or reconstructed $K^{*}$ mass if applicable) of each candidate event to extract the signal yields. As an illustration, Fig. 1 shows the fit results for $B^{0} \rightarrow \Lambda \bar{\Lambda} K^{* 0}$ in the $M_{\Lambda \bar{\Lambda}}$ region below $2.85 \mathrm{GeV} / \mathrm{c}^{2}$, which we refer to as the threshold-mass-enhanced region.
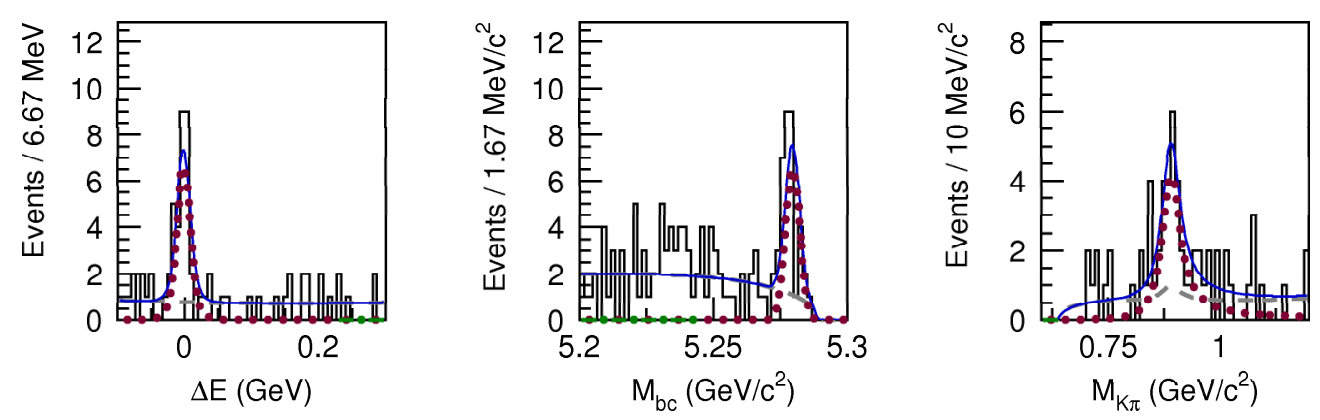

Figure 1: Distributions of $\Delta E$ (with $5.27 \mathrm{GeV} / \mathrm{c}^{2}<M_{\mathrm{bc}}<5.29 \mathrm{GeV} / \mathrm{c}^{2}$ and $0.816 \mathrm{GeV} / \mathrm{c}^{2}<M_{K \pi}<0.976$ $\mathrm{GeV} / \mathrm{c}^{2}$ ), $M_{\mathrm{bc}}$ (with $|\Delta E|<0.05 \mathrm{GeV}$ and $0.816 \mathrm{GeV} / \mathrm{c}^{2}<M_{K \pi}<0.976 \mathrm{GeV} / \mathrm{c}^{2}$ ) and $M_{K \pi}$ (with $|\Delta E|<0.05$ $\mathrm{GeV}$ and $5.27 \mathrm{GeV} / \mathrm{c}^{2}<M_{\mathrm{bc}}<5.29 \mathrm{GeV} / \mathrm{c}^{2}$ ) for $B^{0} \rightarrow \Lambda \bar{\Lambda} K^{* 0}$ in the threshold-mass-enhanced region. The solid curves, dotted curves, and dashed curves represent the total fit result, fitted signal and fitted background, respectively. 
The obtained branching fractions with detection efficiencies determined from Monte Carlo simulation are summarized in Ref. [4]. Comparing with the measured branching fractions for $B \rightarrow p \bar{p} h$ [3], the small value of $\mathscr{B}\left(B^{+} \rightarrow \Lambda \bar{\Lambda} \pi^{+}\right)$and the large value of $\mathscr{B}\left(B^{0} \rightarrow \Lambda \bar{\Lambda} K^{0}\right)$ may indicate that the underlying dynamics of $B \rightarrow \Lambda \bar{\Lambda} h$ are quite different from those of $B \rightarrow p \bar{p} h$. These results also imply that the $\bar{s}$ quark from $\bar{b} \rightarrow \bar{s}$ penguin diagram does not necessarily hadronize to form a $K^{+}$; the probability of forming a $\bar{\Lambda}$ is not negligible.

\section{Results of $B^{+} \rightarrow p \bar{\Lambda} \pi^{+} \pi^{-}$}

The observed charmless baryonic $B$ decays so far are three-body decays only. However, for the charmful case, one interesting hierarchy has been established experimentally, namely $\mathscr{B}\left(B^{0} \rightarrow\right.$ $\left.p \bar{\Lambda}_{c}^{-} \pi^{+} \pi^{-}\right)>\mathscr{B}\left(B^{+} \rightarrow p \bar{\Lambda}_{c}^{-} \pi^{+}\right)>\mathscr{B}\left(B^{0} \rightarrow p \bar{\Lambda}_{c}^{-}\right)[7,8,9]$. We search for the four-body charmless baryonic decay $B^{+} \rightarrow p \bar{\Lambda} \pi^{+} \pi^{-}$with the hope of confirming the hierarchy. We study the mass spectra for $p \bar{\Lambda}, p \pi^{-}, p \pi^{+}, \Lambda \pi^{-}, \Lambda \pi^{+}, \pi^{+} \pi^{-}, p \bar{\Lambda} \pi^{-}, p \bar{\Lambda} \pi^{+}, p \pi^{+} \pi^{-}$and $\Lambda \pi^{+} \pi^{-}$combinations using the $B$ signal yields obtained as functions of those masses. There is a clear enhancement near threshold from the $p \bar{\Lambda}$ mass plot for signal candidates. We fit $p \bar{\Lambda}$ mass with a threshold function and the result is shown in Fig. 2.

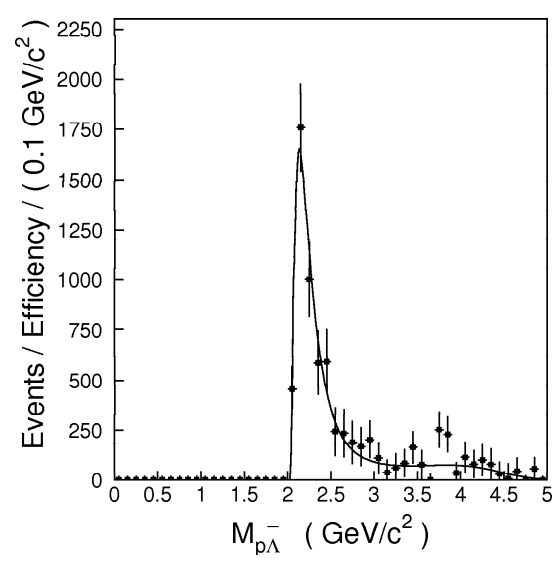

Figure 2: $B$ signal yields obtained as a function $p \bar{\Lambda}$ mass. Here, the yields are corrected for the mass dependent efficiency. The solid curve represents the fit with a threshold function.

We also observe a clear signal of $\rho$ resonance and a hint of $f_{2}$ in the $\pi^{+} \pi^{-}$spectrum. To investigate signals of three body decays like $B^{+} \rightarrow p \bar{\Lambda} \rho$ and $B^{+} \rightarrow p \bar{\Lambda} f_{2}$, we perform an unbinned extended likelihood fit and the fit result is shown in Fig. 3. The resulting signal yields are $167.8_{-23.7}^{+25.0}, 131.2_{-17.5}^{+18.3}$ and $39.1_{-13.96}^{+14.9}$ with statistical significances $9.1,9.5$, and 3.0 for nonresonant $B^{+} \rightarrow p \bar{\Lambda} \pi^{+} \pi^{-}, B^{+} \rightarrow p \bar{\Lambda} \rho$ and $B^{+} \rightarrow p \bar{\Lambda} f_{2}$, respectively. The significance is defined as $\sqrt{-2 \ln \left(L_{0} / L_{\max }\right)}$, where $L_{0}$ and $L_{\max }$ are the likelihood values returned by the fit with the signal yield fixed to zero and at its best fit value, respectively.

The branching fractions are calculated using efficiencies estimated from MC simulations and are listed below: $\mathscr{B}\left(B^{+} \rightarrow p \bar{\Lambda} \pi^{+} \pi^{-}\right)=\left(5.92_{-0.84}^{+0.88} \pm 0.69\right) \times 10^{-6}, \mathscr{B}\left(B^{+} \rightarrow p \bar{\Lambda} \rho\right)=\left(4.78_{-0.64}^{+0.67} \pm\right.$ $0.60) \times 10^{-6}$, and $\mathscr{B}\left(B^{+} \rightarrow p \bar{\Lambda} f_{2}\right)=\left(2.03_{-0.72}^{+0.77} \pm 0.27\right) \times 10^{-6}$. The above results are preliminary. 
(a)

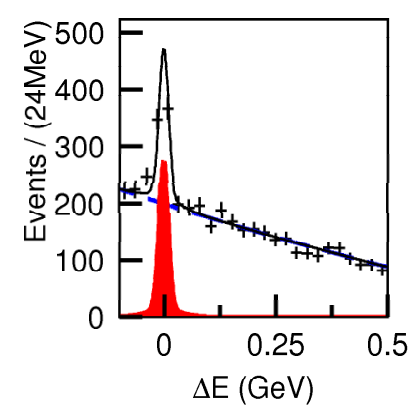

(b)

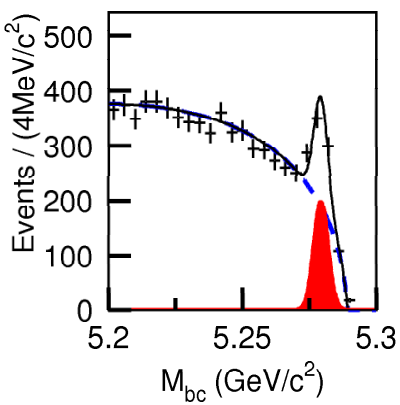

(c)

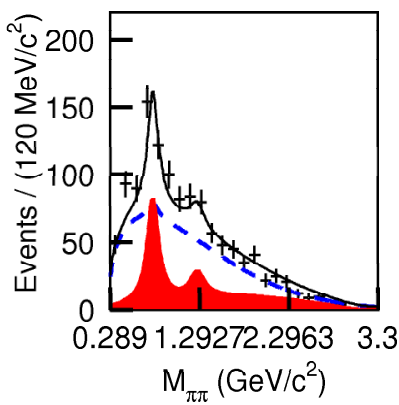

Figure 3: Distributions of (a) $\Delta E$ (with $5.27 \mathrm{GeV} / c^{2}<M_{\mathrm{bc}}<5.29 \mathrm{GeV} / c^{2}$ ), (b) $M_{\mathrm{bc}}$ (with $|\Delta E|<0.05 \mathrm{GeV}$ ) and (c) $M_{\pi^{+} \pi^{-}}$(with $5.27 \mathrm{GeV} / c^{2}<M_{\mathrm{bc}}<5.29 \mathrm{GeV} / c^{2}$ and $|\Delta E|<0.05 \mathrm{GeV}$ ). The solid curve represents the fit projection which is the sum of signal $\left(B^{+} \rightarrow p \bar{\Lambda} \pi^{+} \pi^{-}, B^{+} \rightarrow p \bar{\Lambda} \rho\right.$ and $\left.B^{+} \rightarrow p \bar{\Lambda} f_{2}\right)$ and background (dashed curve) estimations.

This is the first time to observe a four-body charmless baryonic $B$ decay. We also observe low mass $p \bar{\Lambda}$ threshold enhancements for both the $p \bar{\Lambda} \pi^{+} \pi^{-}$and $p \bar{\Lambda} \rho$ modes. The branching fraction of this four-body decay is comparable to the related three-body decays although their central values may indicate that the hierarchy established in the charmful case is still hold.

\section{Acknowledgments}

The author wish to thank the KEKB accelerator group for the excellent operation of the KEKB accelerator. This work is supported by the National Science Council of the Republic of China under the grant NSC-97-2112-M-002-009.

\section{References}

[1] K. Abe et al. (Belle Collaboration), Phys. Rev. Lett. 88, 181803 (2002).

[2] Throughout this report, inclusion of charge conjugate mode is always implied unless otherwise stated.

[3] C. Amsler et al. (Particle Data Group), Phys. Lett. B667, 1 (2008)

[4] Y.W. Chang et al., (Belle Collaboration), Phys. Rev. D79 , 052006 (2009).

[5] A. Abashian et al. (Belle Collaboration), Nucl. Instr. and Meth. A479, 117 (2002).

[6] S. Kurokawa and E. Kikutani, Nucl. Instr. and Meth. A499, 1 (2003) and other papers included in this Volume.

[7] X. Fu et al. (CLEO Collaboration), Phys. Rev. Lett. 79 , 3125 (1997).

[8] N. Gabyshev et al. (Belle Collaboration), Phys. Rev. Lett. 97 , 242001 (2006).

[9] N. Gabyshev et al. (Belle Collaboration), Phys. Rev. D66 , 091102R (2002). 